\title{
Remote-Delivered Services During COVID-19: A Mixed-Methods Survey of College Counseling Center Clinicians
}

Emily Hersch, BA, ${ }^{a+}$ Katherine A. Cohen, $\mathrm{MA}^{\mathrm{b}+*}$, Anjay Saklecha ${ }^{\mathrm{b}}$, Kofoworola D.A. Williams, $\mathrm{PhD}^{\mathrm{b}}$, Yuxi Tan, $\mathrm{MD}^{\mathrm{c}}$, and Emily G. Lattie, $\mathrm{PhD}^{\mathrm{b}}$.

${ }^{a}$ Department of Psychiatry \& Behavioral Sciences, Northwestern University, Chicago, IL, USA;

${ }^{b}$ Department of Preventive Medicine, Northwestern University, Chicago, IL, USA;

${ }^{c}$ Department of Psychiatry, The Second Xiangya Hospital, Central South University, Changsha, China;

${ }^{+}$Indicates equal co-authorship;

*Department of Preventive Medicine, Northwestern University, 750 N Lake Shore Drive, $10^{\text {th }}$ Floor, Chicago, IL 60611, USA. Katherine.Cohen@ Northwestern.edu 


\title{
Remote-Delivered Services During COVID-19: A Mixed-Methods Survey of College Counseling Center Clinicians
}

\begin{abstract}
Objective: The COVID-19 pandemic has drastically increased use of remotedelivered mental health services. This study identifies advantages and limitations of remote-delivered services on college campuses to inform mental health delivery post-pandemic. Methods: Clinicians $(n=30)$ were asked to evaluate COVID-19's impact on their work, environment, and wellness in an online survey. Qualitative data was coded using a thematic analysis approach, while quantitative data was analyzed using descriptive statistics. Results: Many clinicians reported benefits of remote services, including increased accessibility, greater convenience, no change in therapeutic alliance, and decreased stress for clinicians. Clinicians also experienced challenges such as social isolation, technological difficulties, and personal/family concerns. Clinicians envisioned a hybrid service combining online and in-person activities post-pandemic.
\end{abstract}

Conclusions: Overall, remote-delivered mental health services on college campuses have potential in increasing treatment quality while highlighting a necessity for further research in hybrid mental health delivery.

Keywords: College students, mental health, clinicians, telehealth, technology, COVID-19

\section{Introduction}

Mental health service utilization has increased over the last decade on college campuses such that college counseling centers often suffer from long waitlists and a shortage of providers. ${ }^{1}$ Both college students and college counseling clinicians have recognized in previous research studies that delivering mental health support resources to students outside of typical face-to-face services could help to address these concerns. ${ }^{2}$

Remote-delivered services may offer multiple benefits compared to in-person services and increase the ability of clinicians to offer mental health services to individuals who need them. Previous research indicates that mental health clinicians recognize the potential for remote-delivered services to increase access to care, offer 
flexibility in scheduling, and reduce costs for patients. ${ }^{3}$ Yet prior to the COVID-19 pandemic, mental health clinicians delivered approximately seven percent of their services remotely. ${ }^{4}$ This lack of uptake may be related to clinicians' perceptions of the barriers to delivering services remotely. In an online survey conducted prior to the pandemic, clinicians reported concerns around privacy, building rapport, and remote crisis management. ${ }^{3}$ Additionally, while multiple clinical trials have established the efficacy of remote-delivered services, there is little research examining the provision of such services in routine clinical practice. ${ }^{5,6}$

In response to the rapid spread of the COVID-19 virus, mental health providers quickly shifted to delivering nearly eighty-five percent of their services remotely during the pandemic. ${ }^{4}$ This shift to mostly online-delivered services offered an unanticipated opportunity to build on previous research examining nontraditional service delivery. Many researchers have suggested that this shift will ultimately improve mental health service delivery, as it will allow clinicians and researchers to re-evaluate how to provide services that are both accessible and scalable. ${ }^{7}$

The switch to remote-delivered mental health services is especially crucial to investigate on college campuses, where students report multiple barriers to receiving mental health services, including long waitlists, inaccessible locations, and limited office hours. ${ }^{2}$ Such challenges to obtain services may pose risks to student well-being as mental health concerns often go untreated. Many health and academic benefits of counseling service utilization have been cited in previous research studies such as improved retention rates and wellbeing, emphasizing the growing need for accessible mental health care on college campuses. ${ }^{8-10}$

This need for mental health services was exacerbated during the COVID-19 pandemic, as college students reported rising rates of distress and more difficulty 
accessing mental health services. ${ }^{11-13}$ These rising concerns around student mental health during the lockdown were met through a shift to remote-delivered services on college campuses across the nation, offering an opportunity to examine clinicians' perceptions of the benefits and limitations of providing remote services to college students.

This shift additionally offered an unprecedented opportunity to examine how remote-delivery could impact staff wellness and clinician burnout. Burnout among mental health clinicians is historically high and can lead to adverse outcomes such as physical and mental health concerns, job dissatisfaction, negative attitudes towards clients, and high staff turnover. ${ }^{14,15}$ During the pandemic mental health professionals face heightened risk of experiencing increased workload, stress and job demands, and lack of confidence in delivering remote services, yet it remains unknown how remote work impacted clinicians' wellbeing during the pandemic. ${ }^{16}$ Therefore, more research is needed to understand clinicians' experiences delivering remote telehealth services during COVID-19 and the impact it had on their personal wellness and job satisfaction. Understanding college counseling center clinicians' perceptions of telehealth delivery during the COVID-19 pandemic is critical to the future of college student mental health service delivery. The adoption of telehealth services post-pandemic will likely be impacted by how clinicians viewed treatment efficacy, accessibility, and staff wellness during the pandemic. While previous research has examined clinician attitudes towards delivering telehealth, the authors are not aware of any studies that have investigated college counseling center clinicians' attitudes towards remote-delivered services or the perceived challenges and benefits of delivering remote services during the COVID-19 pandemic. ${ }^{17,18}$ In fact, few studies prior to the onset of the pandemic have looked at telehealth services on college campuses. While there has been limited 
evidence to show the acceptability of telehealth services by college students and mental health providers, further research is needed. This study aims to bridge this gap by examining the benefits, challenges, and acceptability of remote-delivered services among campus counseling clinicians and their willingness to engage in remotedelivered services post-pandemic. ${ }^{19}$

\section{Methods}

\section{Participants}

We used data collected from clinical staff members employed at college counseling centers during July and August of 2020. A total of 30 clinicians from six college counseling centers (ranging from community colleges to four-year colleges and universities) in the Midwest region of the United States participated in the survey. There was a wide variety in the size of colleges and universities - the largest institution represented serves more than 20,000 students per year while the smallest institution represented serves less than 2,000 students per year.

The study team first reached out to the directors of the counseling centers at local schools to invite their staff to participate in the study. After the directors agreed to participate, the clinicians from their staff were recruited via email and presentations at staff meetings. Email addresses were used to verify clinicians' employment status. All study procedures were approved by the authors' Institutional Review Board before enrolling participants.

\section{Survey}

Clinicians were asked to complete an online survey hosted on REDCap that was designed by study staff at Northwestern University. The survey asked clinicians 
questions regarding demographics, the impact of COVID-19 on their experiences in working with students, on their work environment, and on their personal and social wellness, as well as suggestions for future changes in the counseling center workflow as a result of COVID-19. These questions were structured in two formats: multiple choice questions (e.g., "Are students more or less likely to show up for sessions when sessions are delivered remotely?") and open-ended questions (e.g., "If you had initial fears about switching to remote delivered services, what were those fears?"). The full item list is included in Supplementary Materials.

\section{Data Analyses}

Descriptive statistics were used to characterize the study sample and summarize answers to multiple choice questions. Qualitative data collected from open-ended questions were coded by authors using a thematic analysis approach. ${ }^{20}$ Qualitative data was examined both by counseling center and in aggregate. There were no notable differences in the responses of participants by counseling center, so qualitative data analyses were conducted in aggregate. Coders first reviewed the data for thematic content and created a codebook with the primary themes that they identified. After the codebook was created, coders reviewed the transcripts a second time to ensure the accuracy of the codebook and complete a final round of coding. The coders met regularly throughout the analytic process to discuss these codes and ensure validity.

\section{Results}

\section{Participant Characteristics}

A total of 10 college counseling center directors were invited to have their staff participate in this study, and 6 college counseling center directors agreed to have their 
center participate. Response rates within counseling centers ranged from $35 \%$ to $100 \%$. There was a total of 30 participants between the ages of 29 and $65(M=44.22, S D=$ 9.63). The majority of participants indicated their gender was "female" $(76.7 \%)$ with the remainder indicating their gender was "male." The sample was predominantly white (80\%) and non-Hispanic (90\%). Participants held a variety of job titles, including psychologists $(60 \%)$, social workers (20\%), psychiatrists $(3.3 \%)$, and "other" job titles (16.70\%). Job titles listed under "other" included "clinical counselor" and "crisis manager." Participants had been working at their college counseling centers between 0.5 and 18 years $(M=6.38, S D=4.27)$.

\section{Theme 1: Benefits and Challenges of Clinicians Working from Home}

The COVID-19 pandemic necessitated that clinicians work from their homes, providing remote-delivered mental health services. Clinicians were asked about how working from home impacted work-related stress, job satisfaction, and productivity. There was a considerable lack of consensus among clinicians, with multiple clinicians disagreeing over whether they saw increases, decreases, or a lack of change in these areas (see Table 1). Overall, however, slightly more clinicians felt that work-related stress had decreased, job satisfaction had increased, and productivity had increased.

Qualitative results helped to clarify why certain clinicians may have felt dissimilarly about working from home depending on personal differences such as family life, health conditions, or technological equipment. Clinicians identified multiple benefits and limitations of working from home (see summary in Table 2).

\section{Benefits}

Many clinicians noted that because they no longer had to commute to work, they saved money and had more free time. Participant 10 commented, "I get more sleep/am better 
rested because I can skip the commute. It's nice to spend less on gas. ” Participant 14 suggested that not commuting has helped them be able to rise to the increased demand of pandemic era work: "I feel like there has been so much to do, that if I had to commute as well I could not have gotten it all done." No longer commuting to work was especially beneficial to clinicians who had chronic health concerns, as Participant 3 explained:

"I have chronic health issues and being at home has increased my flexibility and decreased the amount of time I am out sick... It is often difficult for me to physically move and being able to work from home allows me to meet with clients without having to go out, drive, go up stairs, etc."

Similarly, some clinicians reported that their balance between work and personal activities had improved because of their ability to take more breaks, multitask, or spend time with family members while working from home. Participant 10 commented, "I like being able to move/walk around during phone sessions. My work/life balance has improved, since I can run the laundry or the dishwasher during sessions rather than having to cram such chores into my small amount of free-time.” For many clinicians, working from home allotted them the flexibility to engage in their work in a meaningful way, despite the many challenges of providing mental health care during the pandemic.

\section{Challenges}

Clinicians' reported challenges to delivering telehealth services were divided into social concerns, personal concerns, and technological concerns.

Social Concerns. Due to the limits of working from home, clinicians reported experiencing feelings of isolation and a decrease in informal communication between co-workers. Participant 5 reported feeling "more distant and disconnected by the missed 
opportunities for informal chats in doorways while walking back and forth." Similarly, Participant 18 felt that their "biggest challenge is missing the connection, camaraderie with coworkers through those informal interactions at the doorway, water cooler, and (most importantly) coffee station." They further explained that they "can't seem to recreate those (connections) via video check-in.” Many clinicians indicated that they meet as a group to check-in on staff wellness on a regular basis $(66.7 \%, 20 / 30)$ and that they feel supported by their clinic during the COVID-19 pandemic $(83.30 \%, 25 / 30)$. Clinicians, such as Participant 23, suggested that "regular check-ins and space for social contact with staff members is helpful," but cannot replace face-to-face interaction.

Personal concerns. Personal concerns such as physical discomfort and balancing home life were seen as impacting clinicians' ability to focus on telehealth sessions and manage their time. Multiple clinicians reported feeling "exhaustion from being on the screen all the time" (Participant 2). Clinicians reported eye strain from looking at screens and physical discomfort from sitting without substantial movement throughout the day. Balancing home life, including childcare, was also a major concern among clinicians. One-third $(n=10)$ of clinicians indicated that they are having a more difficult time than usual balancing their work and home life. Participant 5 expressed that, "It's very hard to have split attention as a working parent...working with young kids in the home makes it impossible to be fully focused/immersed in work for prolonged periods of time." Participant 23 succinctly summed up these concerns by stating, "It's hard to be a parent and a therapist under the same roof." Other distractions related to home life also made it difficult to work, such as noises from neighbors or another member of the household working from home. Clinicians without a private office space dedicated to work expressed more difficulty conducting therapy sessions. 
Additionally, clinicians reported that there was less mental separation between work and personal activities when working from home. Participant 13 suggested that this concern made them less productive: "I associate home with relaxation so it's sometimes hard to find the drive to want to do a lot of work when I'm at home... also hard to separate work hours from non-work hours. " On the other hand, Participant 21 suggested that it led them to overwork themselves: "I have less boundaries between work and home and end up working late into the evening and on weekends when I normally wouldn't do that as much." Clinicians indicated that these concerns added additional stress to the work environment.

Technological concerns. Clinicians had a significant amount of technical difficulties, including internet connectivity problems and a lack of proper equipment (desktops, monitors, etc.). Internet connectivity problems were persistent across clinicians from multiple universities. Participant 16 reported that they experienced "internet dysconnectivity at the worst possible times on about 5 occasions. " Internet speed concerns were exacerbated for clinicians who lived with multiple other people using the same WiFi within their household to work or complete online learning. Multiple clinicians reported spending personal money to upgrade their internet package or to buy new computers. Many clinicians agreed that their universities or colleges should provide their staff with computers and high-speed internet so they would not have to rely on their own equipment.

\section{Theme 2: Accessibility of Remote-Delivered Services}

Clinicians were asked about how the accessibility of services changed as a result of online delivery. There was an even split of clinicians who reported that accessibility had either increased or decreased. 36.7\% (11/30) of clinicians across universities reported 
that remote-delivered counseling center services increased students' access to mental health care, while $36.7 \%$ (11/30) reported that remote-delivered counseling center services decreased students' access to mental health care. Both sets of clinicians brought forward important factors to consider regarding when, how, and for whom remotedelivered services could make mental health treatment more or less accessible. For example, some clinicians felt that online service delivery was an effective way to mitigate barriers students typically face in accessing in-person counseling services such as long commutes, transportation issues, or privacy concerns. Participant 10 explained, "students have greater access to our service because they do not have the same barriers to getting to our office as they otherwise would have." Many clinicians saw telehealth as a way to offer mental health care to students who may experience barriers to accessing traditional services.

Clinicians also proposed that online delivery could extend the reach of services to students who typically might not have engaged with the counseling center. Participant 24 said, "I can imagine that students who might not have normally come into the counseling center might be able to access our services easier online." Clinicians suggested that increased student engagement may be in part due to greater convenience experienced by students when accessing services. Participant 18 explained that it is "easier to log in than to make sure one is physically in the Center." Overall, “students appear to enjoy the convenience” (Participant 8).

According to counseling center clinicians, increased flexibility in scheduling also contributed to greater accessibility for students. Some clinicians started working alternative hours during the switch to remote delivered services rather than working their standard business operating hours. This allowed students to access services outside of traditional business hours and at times that worked better for their schedules. 
Participant 18 remarked that, "this delivery system offers some flexibility for many of our students in terms 'fitting in' appointments around other responsibilities in a way that would be impossible at the Center." Clinicians felt that "student access to services is increasing in our office by having evening and weekend hours available" (Participant 4).

While many clinicians saw the potential for remote-delivered services to increase students' access to and engagement with mental health care, other clinicians raised concerns over students' access to resources that would enable them to appropriately utilize these services. For some clinicians, it was particularly challenging to work with students for whom privacy was a concern. Participant 23 expressed that "it has been more difficult to treat some students with family concerns, as finding privacy at home for them has been difficult." This was also highlighted by a clinician who reported that the quality and depth of their sessions suffered as a result of privacy concerns. Participant 21 shared:

"I have a client who is in a one-bedroom apartment with her mother and brother, and therapy often revolves around her unstable relationship with her mother. She emails me with potential times to meet about every 2-3 weeks when her mother is going to be at work. We often do phone sessions and she sometimes just goes for a walk because of the lack of privacy. We end up doing much more case management than deep therapy because of her current situation."

For some clinicians, students' lack of privacy limited the accessibility of remote delivered services. In addition to concerns over students' access to a private space, clinicians also reported how limited access to technology and a reliable internet connection was a common issue for students. These issues resulted in repeated disruptions to counseling services. Despite these concerns, clinicians generally 
remained hopeful about the potential of remote-delivered services to increase the accessibility of mental health care for college students. Participant 26 shared:

\begin{abstract}
"I believe we can have dedicated staff who provide remote telemental health services and staff who provide in-person services as well...this model of service provision could really help a lot of folks if we also take into consideration the necessity to advocate for the larger community to have access to a working laptop and internet connection regardless of ability to pay for these services."
\end{abstract}

Clinicians emphasized the importance of providing students with tools that would enable them to effectively engage in remote counseling services.

\title{
Theme 3: Therapeutic Alliance
}

The overwhelming majority of clinicians expressed initial concerns over whether a strong therapeutic alliance could be maintained over telehealth appointments. First, telehealth appointments were seen as limiting the clinicians' ability to obtain clinical information by observing their clients' physical and emotional behavior. Participant 3 expressed: "I was concerned about not being able to see the client as well and losing information I usually gain through observation such as body language.” Similarly, when a telehealth appointment ended, it could be difficult to gauge the effects of the session on the client. Participant 21 noted: "I can't tell how beneficial a session was for a client without asking because I can't get as solid of a sense of their emotional expression." Second, clinicians were, "worried about the level of connection that would be possible (Participant 24)" when establishing initial rapport with clients. Participant 3 described this experience: "I tend to feel more anxious meeting with people, especially new clients. It takes me longer to feel like I know someone." Lastly, clinicians expressed uncertainty about the "depth" of the work they could do remotely, as Participant 5 noted: "Doing more in-depth work has been hard without physical presence." Overall, 
clinicians were worried about feeling disconnected to their clients.

Some clinicians expressed that their initial fears around being unable to establish and maintain therapeutic alliances were relieved and connecting with clients worked better than they anticipated. When asked about how therapeutic alliance changed as a result of switching to remote-delivered services, $76.7 \%$ (23/30) of clinicians believed therapeutic alliance had not changed, 16.7\% (5/30) believed it had increased, and only $6.7 \%(2 / 30)$ believed it had decreased. Participant 20 commented that, "The connection is different, but still there and powerful." A subset of clinicians indicated that delivering services from home made them more open with clients and the mutual experiences related to the COVID-19 pandemic had improved their therapeutic alliances. Participant 10 believed that:

"Alliance has increased because we now implicitly share this common bond of being impacted by COVID. I also think I'm more human to clients now that they can see my home and very occasionally hear an errant squeal from my daughter in the background."

Additionally, one clinician indicated that remote-delivered services may not carry the same distractions that can take place in the office setting which can promote connection. Participant 26 indicated, "It has worked for a positive connection with clients because I am able to follow up with them one-on-one without the interruption of other students or employees walking into the office space. I can take messages and follow up. " Despite these potential benefits, some clinicians maintained that face-toface interactions were preferable for maintaining therapeutic alliance because, "In person sessions feel more connecting” (Participant 20).

\section{Theme 4: Additional Benefits and Challenges of Remote-Delivered Services}

Initial reaction to remote-delivered services 
Clinicians reported their initial reaction to the switch to remote-delivered services. While few clinicians felt "very negative/very pessimistic" $(3.3 \%, 1 / 30)$, a considerable number of clinicians felt "negative/pessimistic" $(23.3 \%, 7 / 30)$ or "neutral" $(26.70 \%$, 8/30) about the transition to remote services. However, the majority of clinicians felt positive/optimistic $(30.00 \%, 9 / 30)$ about the switch, with some clinicians reporting they felt "very positive/optimistic $(16.7 \%, 5 / 30)$.

\section{Benefits}

Clinicians reported many benefits associated with remote services such as increased therapy adherence, attendance, and engagement (see Table 3). Most clinicians (86.7\%, 26/30) reported that adherence to treatment had stayed the same or increased due to the switch to remote-delivered services. Participant 10 noted how,

"Students seem to have fewer barriers to treatment adherence and tend to stick around for the entirety of the treatment plan. They also seem to seldom feel that they need a "break" from therapy as they sometimes do when life is busier."

Participant 26 explained, "students are able to log on with ease (granted they have consistent internet connection) to connect with their counselor. I believe this allows students to adhere to a routine." Clinicians also reported that there were fewer campus-related activities competing for students' attention, leading to increased attendance and less cancelations. Participant 10 stated, "I almost never have a cancellation or no-show anymore, seemingly because students have less competing for their time." Almost all of the clinicians $(93.3 \%, 28 / 30)$ felt that that attendance was unchanged or improved as a result of remote-delivered services in the pandemic. Clinicians also reported that students' engagement with services had increased as 
"students have been more likely to engage in weekly therapy when we previously were not able to offer this as much" (Participant 21).

Clinicians also reported that remote-delivered services could reduce stigma for students accessing mental health care. While students would traditionally access these services from the counseling center, they could now engage with counseling services from their homes. Clinicians shared that, "meeting remote can feel more comfortable for clients who are impacted by stigma related to counseling" (Participant 19).

Although not all students benefited from accessing services remotely, one clinician reported that in some situations there may be "increased privacy for students living at home" (Participant 17).

Additionally, clinicians noted that remote-delivered care could be as effective as in-person services, and for some students lead to even better clinical outcomes. In particular, some clinicians suggested that students with certain presentations, such as anxiety, may be better suited for remote-delivered services. Participant 11 noted "that some clients actually connect better virtually and can open up more" and virtual delivery "reduced the anxiety of sitting face to face in a room with someone." This was supported by other clinicians who reported that "my socially anxious students preferred remote sessions and were able to better engage in them" (Participant 26). Many clinicians felt that remote-delivered services were effective and even preferred by some students who "embraced the switch to telehealth" (Participant 15). Participant 9 reflected on the future of remote-delivered services for college students, "I think this is where the field is heading. Some may be uncomfortable with the idea overall while some may have discomfort and with time, will experience therapeutic changes regardless of delivery."

Challenges 
Although clinicians identified many advantages to delivering telehealth, some clinicians expressed concerns around the ethical and legal issues associated with remote delivery (see Table 3). Clinicians expressed frustration around the limitations of working with students from out of state as "state licensure laws and recommendations from the College's legal council have made it not possible for us to provide therapy to out of state students" (Participant 21). Clinicians also expressed concerns around crises and hospitalization procedures. Participant 10 shared, "I worried I wouldn't know what to do if a crisis occurred while I was on a remote session with a student, or worse, in a group." Some clinicians felt that these crisis procedures were more difficult to follow remotely than in-person.

Many clinicians also identified specific clinical presentations that they felt made it more difficult to provide students with remote-services. Clinicians felt online counseling services may not be appropriate for all students as "some clients don't seem to translate to virtual delivery as well as others" (Participant 5). Participant 13 stated that, "more serious risk concerns/presentations have been harder to manage remotely, aka, substance abuse, SI, etc." Other clinicians shared this difficulty and felt that, "trauma work and more complex presentations are harder to manage remotely" (Participant 19). Clinicians also found it difficult to support students who may be feeling isolated due to the pandemic. Participant 10 remarked, "it's also overall more difficult to help students who feel isolated or are having difficulty making friends, given the impact of COVID." Clinicians were increasingly "worried that students felt disconnected from campus community" and feared that "reaching out to Wellness Center for support did not seem like an option” (Participant 18).

Lastly, some clinicians felt like online treatment was not as effective as inperson services. Participant 16 indicated they were, "still able to provide effective 
treatment to clients, although it is not the same (e.g., as effective) as in-person treatment" and was preferential towards in-person delivery because, "in my strong opinion, remote services will never be as effective of a treatment for individual counseling or process groups as compared to in-person treatment." Participant 21 also stated, "I don't feel like I am as effective in remote therapy as I am in the office with clients."

Clinician preferences for future service delivery

Despite these challenges, when clinicians were asked about their preferences for how services should be delivered for the remainder of the pandemic, the majority of clinicians reported wanting to deliver exclusively remote services $(86.7 \%, 26 / 30)$, with only a few clinicians wanting to offer in-person services as well $(13.3 \%, 4 / 30)$.

\section{Hybrid Delivery Model}

Clinicians were also asked about their preferences for service delivery after the COVID19 pandemic. Almost all clinicians wanted to continue to offer remote-services to students, typically as part of a hybrid-model including both in-person and virtual delivery options. The vast majority $(96.7 \%, 29 / 30)$ of clinicians reported wanting to offer some form of remote services after the COVID-19 pandemic. The most commonly reported preference $(90 \%, 27 / 30)$ was to deliver either an even split of services or primarily in-person services with remote services as optional. A minority of clinicians indicated they would like to deliver exclusively in-person $(3.3 \%, 1 / 30)$, exclusively remote $(3.3 \%, 1 / 30)$, or primarily remote services with in-person services as optional $(3.3 \%, 1 / 30)$. Overall, clinicians emphasized the importance of staying flexible and offering students a choice in service delivery. Participant 2 hoped their counseling center would have the "flexibility to offer hybrid services to students based on client's 
needs." One clinician shared, "The more options we can offer students, the more likely they are to access services" (Participant 3). Clinicians also felt it was important to continue to offer in-person services. Participant 26 expressed that, "others may want to return to face-to-face which I also believe should be offered as well." Clinicians felt it could be useful to provide recommendations about which modality might be better suited for a particular student, "I think students will be given the option of remote or inperson services when meeting for an initial consultation. Therapists can also give recommendations about which modality based on client presentation" (Participant 28). Despite wanting to continue to offer in-person services, clinicians recognized that a remote "delivery system will remain popular with [the] student population" postpandemic (Participant 18).

\section{Discussion}

The COVID-19 pandemic has had a significant impact on college students' mental health status and well-being, presenting a number of challenges for college counseling centers. As a response to this, college counseling centers have been delivering mental health services remotely. It is possible that remote-delivered mental health services can overcome common barriers associated with traditional, face-to-face care, such as long waitlists and geographic barriers. Yet, little research has been done to examine the feasibility and efficacy of remote-delivered services through campus counseling centers, especially during the COVID-19 pandemic. This study begins to fill this scientific gap, by examining college counseling center clinicians' perceptions of remote-delivered services during the pandemic. As more of the population is vaccinated and colleges and universities return to normalcy, a better understanding of remote-delivered services and how clinicians perceive this service is crucial to future implementation of mental health service delivery on college campuses. 
In our study, campus counseling center clinicians reported many benefits of remote-delivered care such as increased accessibility, convenience for both clinicians and students, and greater treatment adherence. These findings align with past research showing clinicians' generally positive attitudes towards tele-health. ${ }^{17}$ Our findings also show that despite clinicians' initial concerns, most campus counseling clinicians felt that they were able to develop strong therapeutic alliances with students and believed that online treatment was both effective and efficient. In a narrative review conducted by Simpson \& Reid ${ }^{21}$ researchers found that therapeutic alliance developed through remote-services was comparable to therapeutic alliance developed through in-person sessions as rated by both clinicians and clients. Given the overlap in these results and the findings of the present study, future work may focus on communicating to clinicians that therapeutic alliance in remote-delivered services may not be inferior to that in faceto-face services. Additionally, future work may investigate whether this finding is consistent across different populations or clinical presentations. Overall, additional research is needed to determine for whom remote-delivered services may be most beneficial.

As described in the results, clinicians in this study suggested that students with anxiety appeared to have preferred the remote-delivered sessions over face-to-face sessions and were more comfortable engaging in remote sessions. What remains to be known is whether students with anxiety benefitted from remote-delivered services as much as, or more than, they would have from face-to-face sessions. In a study comparing face-to-face therapy to telephone-delivered therapy for depression, StilesShields and colleagues ${ }^{22}$ found that comorbid anxiety was a differential treatment predictor. They found that there were no differences in therapy outcomes between telephone-based therapy and face-to-face therapy among those without comorbid 
anxiety disorders. However, participants with comorbid anxiety disorders who received telephone-based therapy had significantly higher symptom severity over time compared to their counterparts without anxiety, where there were no differences in outcomes between participants with and without comorbid anxiety disorders who received faceto-face therapy. As clinicians envision the use of telehealth in the post-pandemic world, it will be valuable to understand that comparative effectiveness of face-to-face and remote-delivered mental health services for a variety of mental health concerns so that clinicians may direct students to treatments that are both acceptable to the student and effective for their presenting concerns.

This study provides evidence of college campus counselors' support for hybrid treatments of both remote and in-person mental health service delivery on college campuses. The concept of offering hybrid-treatments align with current findings that mental health treatment will likely be delivered in a blended format post-pandemic. ${ }^{23}$ As such, this crisis may have served as a springboard for beneficial changes for college student mental health. There has been a recent history of students being dissatisfied with the available services offered through counseling centers as they often face long waitlists and a lack of flexibility in scheduling appointments. ${ }^{2}$ As many of the clinicians noted, remote-delivered services enabled students to access care from the convenience of their homes and for some students, led to increased treatment adherence, attendance, and engagement and reduced stigma in accessing care. These findings align well with previous literature indicating that college students are interested in nontraditional mental health tools services as a supplement or enhancement to traditional face-to-face mental health services. ${ }^{24,25}$ Many additional benefits related to staff wellness were also seen. Clinicians reported improved work-life balance, reduced stress, and slight improvements to job satisfaction and productivity. For both clinicians and students, 
remote-delivered services appeared to be well-liked and provided many advantages over face-to-face sessions.

Despite these benefits, participants noted that many challenges of remotedelivered services remain, such as concerns over privacy, internet connectivity, technology access, and ethical and legal considerations. Challenges such as the inability for clinicians to see students across state lines limited the widespread accessibility and efficacy of remote mental health services and should be considered when envisioning mental health services after the pandemic. The APA ${ }^{26}$ has begun to develop guidelines and loosen restrictions around remote-service delivery through college campus counseling centers, highlighting the shift towards and acceptance of remote-delivered mental health services on college campuses. However, additional work must be done in order to ensure that students have access to remote mental health care and the tools and resources necessary to best utilize these services.

In addition, more resources are needed to support clinicians' well-being during remote-service delivery including those that focus on social isolation, increasing interactions with co-workers, and providing equipment that supports working from home such as computers and home offices. Developing protocols and additional guidance on maintaining staff wellness and an optimal work environment for campus counseling clinicians delivering remote mental health services may help to address clinicians' concerns and improve the adoption and delivery of online mental health care to college students.

\section{Limitations}

This study has several limitations which must be considered when interpreting the results. First, while the institutions that chose to participate in this study ranged in size and represented both community colleges and 4-year universities, this study focused on 
campuses from one geographic area in the United States. Second, counseling center directors served as the gatekeepers for study participation, and therefore these results may not be representative of all counseling centers. Lastly, the clinicians included in this sample were mostly female-identifying and non-Hispanic white. Future work may focus on recruiting a more diverse sample of college counseling center clinicians.

\section{Conclusion}

This study advances the current literature on clinician perceptions of remote-delivered services by being the first study to examine the attitudes of campus counseling center clinicians' towards delivering remote mental health services to college students during the COVID-19 pandemic. The results provide initial evidence for the successful delivery of remote-delivered mental health services on college campuses that are accessible outside of face-to-face provision. Additionally, the challenges and limitations reported by clinicians in this study can inform researchers and universities of the areas that will need to be addressed if remote-services are to be delivered on a wider scale. By understanding college counseling center clinicians' response to remote-delivered services, researchers and administrators can work towards designing remote-mental health services that are effective, accessible, and tailored to college students' needs postpandemic.

\section{Acknowledgements}

This work was supported by a grant from the National Institutes of Health (K08 MH112878).

\section{Declaration of Interest Statement}

The authors have no conflicts of interest to report. 


\section{References}

1. Lipson S.K., Lattie E.G., Eisenberg D. Increased rates of mental health service utilization by U.S. college students: 10-year population-level trends (2007-2017). Psychiatr Serv. 2019;70(1):60-63.

2. Cohen K.A., Graham A.K., Lattie E.G. Aligning students and counseling centers on student mental health needs and treatment resources. Journal of American College Health. 2020;1-9.

3. Perry K, Gold S, Shearer E.M. Identifying and addressing mental health providers' perceived barriers to clinical video telehealth utilization. Journal of Clinical Psychology. 2020;76(6):1125-1134.

4. Pierce B.S., Perrin P.B., Tyler C.M., McKee G.B., Watson J.D. The COVID-19 telepsychology revolution: A national study of pandemic-based changes in U.S. mental health care delivery. The American Psychologist. 2021;76(1):14-25.

5. Wagner B, Horn AB, Maercker A. Internet-based versus face-to-face cognitivebehavioral intervention for depression: a randomized controlled non-inferiority trial. $J$ Affect Disord. 2014;152-154:113-121.

6. O'Reilly R, Bishop J, Maddox K, Hutchinson L, Fisman M, Takhar J. Is telepsychiatry equivalent to face-to-face psychiatry? Results from a randomized controlled equivalence trial. Psychiatr Serv. 2007;58(6):836-843.

7. Moreno C, Wykes T, Galderisi S, et al. How mental health care should change as a consequence of the COVID-19 pandemic. The Lancet Psychiatry. 2020;7(9):813-824.

8. Lee, D, Olson, EA, Locke, B, Michelson, ST, Odes, E. The effects of college counseling services on academic performance and retention. Journal of College Student Development. 2009;50(3):305-319.

9. Huenergarde, M, College students' well-being: use of counseling services. American Journal of Undergraduate Research, 2018;15(3).

10. Vescovelli, F, Melani, P, Ruini, C, Ricci Bitti, PE, Monti, F. University counseling service for improving students' mental health. Psychological services, 2017;14(4):470480.

11. Son C, Hegde S, Smith A, Wang X, Sasangohar F. Effects of COVID-19 on college students' mental health in the United States: Interview Survey Study. J Med Internet Res. 2020;22(9):e21279.

12. Wang X, Hegde S, Son C, Keller B, Smith A, Sasangohar F. Investigating mental health of US college students during the COVID-19 pandemic: cross-sectional survey study. J Med Internet Res. 2020;22(9):e22817.

13. The Healthy Minds Network. The impact of COVID-19 on college student wellbeing. 2020. 
https://www.acha.org/documents/ncha/Healthy_Minds_NCHA_COVID_Survey_Repor t_FINAL.pdf

14. Simionato GK, Simpson S. Personal risk factors associated with burnout among psychotherapists: A systematic review of the literature. Journal of Clinical Psychology. 2018;74(9):1431-1456.

15. Morse G, Salyers MP, Rollins AL, Monroe-DeVita M, Pfahler C. Burnout in mental health services: a review of the problem and its remediation. Adm Policy Ment Health. 2012;39(5):341-352.

16. Joshi G, Sharma G. Burnout: A risk factor amongst mental health professionals during COVID-19. Asian J Psychiatr. 2020;54:102300. doi:10.1016/j.ajp.2020.102300.

17. Reay, RE, Looi, JC, Keightley, P. Telehealth mental health services during COVID19: summary of evidence and clinical practice. Australas Psychiatry, 2020;28(5):514516. https://doi.org/10.1177/1039856220943032

18. Connolly, SL, Miller, CJ, Lindsay, JA, Bauer, MS. A systematic review of providers' attitudes toward telemental health via videoconferencing. Clinical Psychology: Science and Practice, 2020;27(2). https://doi.org/10.1111/cpsp.12311.

19. Khasanshina, EV, Wolfe, WL, Emerson, EN, Stachura, ME. Counseling centerbased tele-mental health for students at a rural university. Telemed $J$ E Health, 2008;14(1):35-41. https://doi.org/10.1089/tmj.2006.0038.

20. Braun V, Clarke V. Using thematic analysis in psychology. Qualitative Research in Psychology. 2006;3(2):77-101.

21. Simpson, SG, Reid, CL. Therapeutic alliance in videoconferencing psychotherapy: a review. Aust J Rural Health, 2014;22(6):280-299. https://doi.org/10.1111/ajr.12149.

22. Stiles-Shields C, Kwasny MJ, Cai X, Mohr DC. Comorbid anxiety as a differential treatment predictor for telephone versus face-to-face administered cognitive behavioral therapy for depression. Depress Anxiety. 2014;31(11):934-940.

https://doi:10.1002/da.22323.

23. Bierbooms, J, van Haaren, M, WA, IJ, de Kort, YAW, Feijt, M, Bongers, IMB. Integration of online treatment into the "New Normal" in mental health care in postCOVID-19 times: Exploratory Qualitative Study. JMIR Form Res, 2020;4(10):e21344. https://doi.org/10.2196/21344.

24. Hadler NL, Bu P, Winkler A, Alexander AW. College student perspectives of telemental health: a review of the recent literature. Current Psychiatry Reports. 2021;23(2):6.

25. Lungu A, Sun M. Time for a change: college students' preference for technologymediated versus face-to-face help for emotional distress. Telemedicine and E-Health. 2016;22(12):991-1000. 
26. APA. College Mental Health, Telepsychiatry: Best Practices, Policy Considerations \& COVID-19. 2020. 


\section{Tables}

Table 1. Changes in Work-Related Stress, Job Satisfaction, and Productivity.

\begin{tabular}{|c|c|c|c|}
\hline & & Count (n) & $\%$ \\
\hline & Total & 30 & $100 \%$ \\
\hline \multirow[t]{3}{*}{ Work-Related Stress } & Notable decrease & 12 & $40 \%$ \\
\hline & No significant change & 7 & $23.3 \%$ \\
\hline & Notable increase & 11 & $36.70 \%$ \\
\hline \multirow[t]{3}{*}{ Job Satisfaction } & Notable decrease & 7 & $23.3 \%$ \\
\hline & No significant change & 13 & $43.3 \%$ \\
\hline & Notable increase & 10 & $33.3 \%$ \\
\hline \multirow[t]{3}{*}{ Productivity } & Decreased & 6 & $20.0 \%$ \\
\hline & Not changed & 17 & $56.7 \%$ \\
\hline & Increased & 7 & $23.3 \%$ \\
\hline
\end{tabular}


Table 2. Summary of Theme 1: Benefits and Challenges of Clinicians Working from Home.

\begin{tabular}{|c|c|c|}
\hline \multicolumn{2}{|c|}{ Benefits } & $\begin{array}{l}\text { Work/life balance } \\
\text { Reduced stress } \\
\text { More accommodating to chronic health concerns } \\
\text { No commute to work } \\
\text { Greater ability to take care of and be with family } \\
\text { Fewer interruptions from co-workers } \\
\text { Ability to multitask }\end{array}$ \\
\hline \multirow{3}{*}{ Challenges } & $\begin{array}{l}\text { Social } \\
\text { concerns }\end{array}$ & $\begin{array}{l}\text { Social isolation } \\
\text { Lack of informal interactions with co-workers }\end{array}$ \\
\hline & $\begin{array}{l}\text { Personal } \\
\text { concerns }\end{array}$ & $\begin{array}{l}\text { Lacking privacy and space at home for sessions } \\
\text { Physical discomfort } \\
\text { Providing childcare while working } \\
\text { Eye strain } \\
\text { Little separation between work and home life } \\
\text { Distractions from home life }\end{array}$ \\
\hline & $\begin{array}{l}\text { Technological } \\
\text { concerns }\end{array}$ & $\begin{array}{l}\text { Internet connectivity } \\
\text { Lack of equipment (e.g., desktop, monitors) }\end{array}$ \\
\hline
\end{tabular}


Table 3. Summary of Theme 4: Additional Benefits and Challenges of Remote-

Delivered Services.

\begin{tabular}{|l|l|}
\hline Benefits & $\begin{array}{l}\text { Increased therapy adherence, attendance, and engagement } \\
\text { Reduced stigma } \\
\text { Led to better therapeutic outcomes for some students } \\
\text { Perceived to be well-liked by students }\end{array}$ \\
\hline Challenges & $\begin{array}{l}\text { Restrictions on working with out-of-state students } \\
\text { Unclear crisis/hospitalization procedures } \\
\text { Specific presentations may not be as suited for telehealth } \\
\text { Student isolation/disconnection } \\
\text { Concerns regarding treatment efficacy compared to in-person } \\
\text { services }\end{array}$ \\
\hline
\end{tabular}

